\title{
Rapid Routine HIV Testing for Psychiatric Inpatients
}

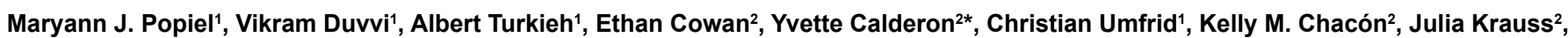

Anuj Rao ${ }^{2}$, Jennifer Zahn' ${ }^{1}$, Uttara Gadde ${ }^{1}$ and Jason Leider ${ }^{2}$

${ }^{1}$ Albert Einstein College of Medicine, Bronx, New York, USA

${ }^{2}$ Jacobi Medical Center, Bronx, New York, USA

\begin{abstract}
The Centers for Disease Control and Prevention recommends that HIV screening be routine and offered in all healthcare settings, including all mental health care facilities. The psychiatric patient population is known to be at higher risk and have significantly higher prevalence of HIV infection than the general population, but remains inconsistently tested and often missed through screening efforts. The objective of this study was to implement and determine the efficacy of a counselor-based HIV screening program on inpatient psychiatric units, and to identify risk factors and barriers to testing within this population. Utilizing dedicated, trained Public Health Advocates, rapid HIV testing was offered to a convenience sample of psychiatric inpatients determined to have capacity to consent. Pre-test counseling was provided and demographic and risk factor data obtained from participants, while reasons for refusal were obtained from those who denied testing. Of consentable patients, $74.6 \%$ were offered testing and $62.9 \%$ of offers were accepted, resulting in 405 patients tested and one new HIV diagnosis. Amongst those who were tested, $26.4 \%$ were found to be high-risk, $33.1 \%$ had a history of drug use and $7.4 \%$ had a history of sex abuse, while $38 \%$ did not receive regular medical care and $22.2 \%$ had never been tested for HIV. The most common reason for patients rejecting testing was the belief that they were not at risk (44\%). In sum, many psychiatric inpatients were high-risk for HIV infection but did not receive regular health care, and personal risk perception is the major barrier preventing patients from obtaining testing. Counselor-based rapid testing proved to be acceptable to psychiatric inpatients and an effective model for screening a large number of high-risk and underserved individuals with minimal added burden to the mental health care team.
\end{abstract}

Keywords: HIV; HIV testing; Psychiatric inpatients; Disease control

\section{Introduction}

The Centers for Disease Control and Prevention (CDC) recommends that opt-out HIV screening be routinely provided in all areas of healthcare, with a minimum of yearly retesting for high-risk patients. These recommendations were made with the goals of early detection, reducing cases of undiagnosed infection, linking patients to treatment, and reducing associated morbidity and transmission to sex partners [1]. Citing similar goals, the American Psychological Association has highlighted the need to prioritize increasing HIV screening in mental health settings [2], and the American Psychiatric Association (APA) has recommended routine HIV screening in psychiatric and substance abuse facilities [3]. However, HIV screening is uncommonly or inconsistently conducted in mental health settings, with rates varying considerably from $17 \%-47 \%$ of patients being tested annually, and $11 \%-89 \%$ of patients never being tested in their lifetime $[4,5]$. HIV testing among the mentally ill increased little following the CDC's most recent recommendations, demonstrating the importance of continued awareness and wider implementation of screening in mental health settings [6].

Routine HIV screening for the psychiatric patient population is of particular importance, as individuals diagnosed with a severe mental illness (SMI) have consistently been found to have infection rates manyfold higher than the general population [7-10], with recent estimates of approximately four-to-eight times greater prevalence $[8,10]$. Per CDC guidelines, high-risk patients are those who inject drugs, engage in sex trade, have multiple sex partners, a partner with HIV or who injects drugs, and men who have sex with men [1]. Patients with SMI more frequently engage in many of these high-risk behaviors compared to those without psychiatric diagnoses, including having multiple sex partners, a history of sexually transmitted infections, engaging in unprotected sex and involvement in sex trade [11]. Additionally, several studies have identified substance abuse as the primary HIV risk factor in the mentally ill $[12,13]$. These patients have dramatically higher rates of substance abuse and injection drug use, frequently associated with sex while intoxicated and with partners who inject drugs [11,14].

In addition to being a high-risk population, many patients with a SMI have significant deficiencies in their knowledge of HIV, while even those who have adequate knowledge are often unable to accurately identify their own level of risk [14-17]. This underscores the necessity of routine HIV screening over targeted, risk-based testing. Offering routine screening to all patients in mental health settings provides an opportunity for counseling and improving patients' knowledge of HIV risk factors and prevention, while potentially identifying many seropositive patients who may be missed in a system dependent on self-identified risk.

Rapid HIV testing offers quick, noninvasive screening easily coupled with counseling, confirmatory testing and linkage to care. Routine, rapid screening has already proven widely effective at detecting many undiagnosed cases of HIV in medical settings [18-21], and linking newly diagnosed patients to care $[21,22]$. The majority of patients who receive an HIV diagnosis have been shown to increase condom use or make additional efforts to lower the risk of transmission [23]. While the individual and public health benefits of improved HIV detection are well documented, studies on the implementation and success of routine screening programs with psychiatric patients remain scarce. Sanger et

*Corresponding author: Yvette Calderon, 3654 Waldo Avenue, Jacobi Medical Center, Bronx, Apt 1a, Bronx, New York 10463, USA, Tel: 917-301-4174; (718) 519-3113; Fax: (718) 519-500; E-mail: ycalderon@gmail.com

Received February 05, 2015; Accepted March 03, 2016; Published March 12 2016

Citation: Popiel MJ, Duvvi V, Turkieh A, Cowan E, Calderon Y, et al. (2016) Rapid Routine HIV Testing for Psychiatric Inpatients. J AIDS Clin Res 7: 555 doi:10.4172/2155-6113.1000555

Copyright: ( 2016 Popiel MJ, et al. This is an open-access article distributed under the terms of the Creative Commons Attribution License, which permits unrestricted use, distribution, and reproduction in any medium, provided the original author and source are credited. 
al. have found routine HIV screening on inpatient psychiatric units to be both practical and acceptable to the majority of eligible patients [24], and rapid testing has been used successfully to identify HIV cases in a variety of mental health settings [10]. However, barriers still exist which make providers hesitant or unable to implement such screening, including lack of time, lack of knowledge required for counseling, other priorities for care, and physician expectations of patient refusal [25].

The objective of this study was to evaluate the use of a streamlined HIV screening program in an inpatient psychiatric setting in an effort to improve and expand routine screening for psychiatric patients. Project B.R.I.E.F (Behavior intervention, Rapid HIV test, Innovative video, Efficient cost and health care savings, Facilitated seamless care) was initially developed to offer efficient routine screening in emergency departments by combining rapid testing with video-assisted education and counseling, electronic information acquisition, and public health advocates to facilitate and link HIV-positive patients to care. Since its initiation, Project BRIEF has been found to be highly acceptable by patients in medical settings and effective at linking newly diagnosed patients to treatment [25]. We sought to expand an adapted version of the program to inpatient psychiatric units to similarly determine screening acceptability and utility in this novel setting, as well as to identify patient barriers to testing, psychiatric diagnoses and risk factor correlates among those tested.

\section{Methods}

\section{Study design}

This was a prospective, descriptive study conducted at Jacobi Medical Center within four psychiatric inpatient units. Testing was offered on the units on 22 days over the course of ten months spanning from July 2013 to July 2014. Per New York City Department of Health and Mental hygiene requirements, demographic and risk factor data were obtained from each participant prior to testing, and psychiatric diagnoses, sex abuse histories and drug abuse histories were obtained post-test via chart review. Protocol was established for conducting western blot confirmatory testing, obtaining CD4+ cell count and viral load from any participant with a positive rapid test, as well as helping these participants complete partner notification forms and linking them to immediate counseling and follow-up care through Jacobi Medical Center.

During testing days on inpatient units, nurses, aids or social workers accompanied individual, eligible patients to a private room on the unit, where each was offered testing by the trained PHA. During the last eight days of testing, acceptability was monitored, and those who denied testing were asked to complete a questionnaire providing a reason for refusal. Participants who accepted testing were provided verbal consent and pre-test counseling by the PHA, and a rapid oral HIV 1-2 antibody test was conducted. After administering the oral swab, the PHA used questionnaires to obtain risk factor data from the participant while awaiting test development. Demographic information including age, sex, race/ethnicity was gathered, along with risk factor assessment, which included those that classify individuals as highrisk per CDC criteria, as well as other risky sex behaviors, sexually transmitted infection histories and drug use and behavioral histories. Additional information was gathered pertaining to the patient's health maintenance, including prior HIV testing and primary care involvement. Results were then read, recorded and discussed with the patient, and any necessary post-test counseling provided.

Psychiatric diagnoses, sexual abuse histories and drug abuse histories were determined by reviewing participants' electronic medical records. Psychiatric discharge summaries were utilized as the primary source of up-to-date DSM-IV-TR diagnoses including primary psychiatric and substance-related diagnoses. Psychiatric medical records were also reviewed for mention of sexual abuse. All information was transferred to a confidential database for SPSS analysis.

\section{Subjects and Recruitment}

Subjects were recruited from inpatient psychiatric units at Jacobi Medical Center, a 500 bed public hospital in the Bronx, NY. A convenience sample of those patients determined to have capacity to consent by the mental health care team was used. Prior to each day of testing, unit psychiatrists and nurses compiled a list of current patients whom they determined to have capacity and to be stable for testing, which was provided to the PHAs. Eligible patients included those who were at least 18-years of age, understood English or Spanish, were medically stable and had capacity. Subjects who had been tested for HIV within the six months prior to this study, did not have capacity or could not understand the consent and counseling process, as well as subjects whom the psychiatric team determined may cause harm to themselves or others due to a new HIV diagnosis were excluded from participation.

\section{Results}

The participants in this study were composed of $59.5 \%$ men and $40.5 \%$ women. All participants were adults, of ages ranging from 18 to 73 -years-old, with a mean age of $37.9 \pm 13.01$ years-old. The majority of participants were racial or ethnic minorities, with $43 \%$ identifying as Hispanic or Latino and $40.2 \%$ identifying as black (Table 1 ). The most common psychiatric diagnoses amongst participants were schizophrenia or other psychotic disorders, which accounted for $65.2 \%$ of the participants, followed by depressive disorders at $15.6 \%$, bipolar disorder at $13.8 \%$, with other diagnoses accounting for the remaining $5.4 \%$ of the participants (Figure 1).

\begin{tabular}{|c|c|c|}
\hline \multirow{2}{*}{ Age } & & $\begin{array}{l}37.86 \pm 13.01 \\
\text { range: } 18-73\end{array}$ \\
\hline \multirow{2}{*}{ Gender } & Male & $241(59.5 \%)$ \\
\cline { 2 - 3 } & Female & $164(40.5 \%)$ \\
\hline \multirow{2}{*}{ Race/ethnicity } & Hispanic/Latino & $174(43 \%)$ \\
\cline { 2 - 3 } & Non-Hispanic Black & $163(40.2 \%)$ \\
\hline \multirow{2}{*}{ HIV tested before } & Yes & $315(77.8 \%)$ \\
\cline { 2 - 3 } & No & $90(22.2 \%)$ \\
\hline
\end{tabular}

Table 1: Demographic Information $(N=405)$.

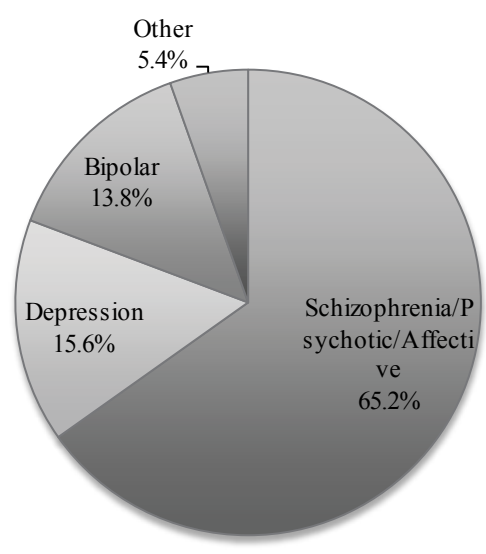

Figure 1: Psychiatric diagnoses $(\mathrm{N}=405)$. 
Citation: Popiel MJ, Duvvi V, Turkieh A, Cowan E, Calderon Y, et al. (2016) Rapid Routine HIV Testing for Psychiatric Inpatients. J AIDS Clin Res 7: 555. doi:10.4172/2155-6113.1000555

Page 3 of 5

During the portion of the study period that acceptability was monitored, $74.6 \%$ of patients determined to have capacity to consent were offered HIV testing (294 out of 394 patients). The remaining $25.4 \%$ of consentable patients were not offered testing due primarily to the unavailability or discharging of patients, or to limitations in time and Public Health Advocate resources. Of the patients offered testing, $62.9 \%$ (185 patients) accepted and one new HIV-positive diagnosis was made (Figure 2). Of the patients who refused testing, a subset was asked to identify their reason for refusal during several of the testing periods (Figure 3). The most common reasons for refusal included the patient feeling that they were not at risk (44.2\%) or having been tested within the last six months (25.6\%), followed by language barrier between the patient and PHA or testing materials (6.97\%), and being afraid that testing would slow psychiatric care or fear of testing itself (each $4.65 \%$ ). Six out of the 43 patients (13.9\%) who were asked for reason for refusal listed their reason as "other," of which five (11.6\%) specified that they had already been diagnosed HIV-positive.

Preventive health and risk factor data was obtained from those who chose to participate and receive HIV testing (Table 2), which showed that $38 \%$ of participants did not receive regular medical care, and $22.2 \%$ had never been tested for HIV in the past. Based on the CDC's five criteria, $26.4 \%$ of participants were found to be high-risk for acquiring HIV: $22.2 \%$ of participants had multiple sex partners, accounting for the most prevalent risk factor among high-risk individuals, $4.9 \%$ had used injection drugs, $4.9 \%$ had engaged in sex trade, $3.2 \%$ had sex with a known injection drug user, and $1.5 \%$ had sex with a known HIVpositive partner. When asked about condom use in the three months prior to testing, $270(66.7 \%)$ of participants said that they never used condoms, 9 (2.2\%) almost never used them and 53 (13.1\%) sometimes did, while only $39(9.6 \%)$ and 32 (7.9\%) participants used condoms "almost every time" or "every time" they had sex, respectively. Fortynine $(12.1 \%)$ of participants had a partner who engaged in sex trade, and $128(31.6 \%)$ used street drugs before intercourse. Due to their relationship with both mental illness and HIV infection, participant psychiatric records were reviewed for substance abuse diagnoses or

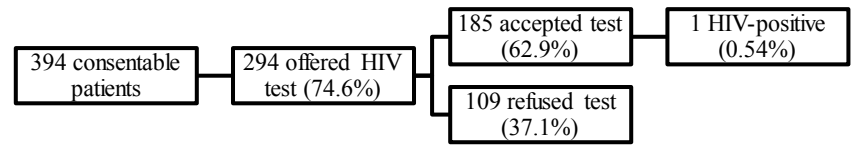

Figure 2: Program acceptability during select study period.

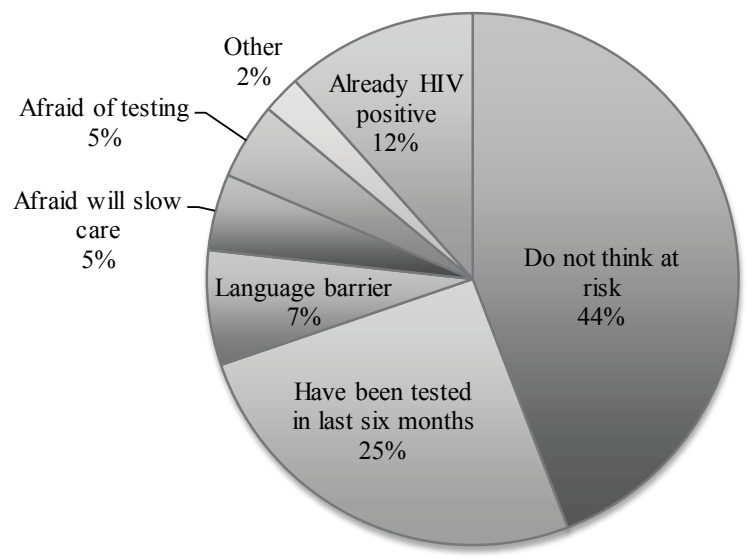

Figure 3: Reasons for refusal of HIV testing $(N=43)$.

\begin{tabular}{|c|c|}
\hline Schizophrenia/Psychotic/Affective & $264(65.2 \%)$ \\
\hline Depression & $63(15.6 \%)$ \\
\hline Bipolar & $56(13.8 \%)$ \\
\hline Other & $22(5.4 \%)$ \\
\hline
\end{tabular}

Table 2: Reasons for refusal of HIV testing $(N=43)$.

\begin{tabular}{|c|c|}
\hline High-risk for acquiring HIV (per CDC criterion) & $107(26.4 \%)$ \\
\hline Multiple sex partners & $90(22.2 \%)$ \\
\hline Injection drug use history & $20(4.9 \%)$ \\
\hline Sex for commodities & $20(4.9 \%)$ \\
\hline Sex with injection drug user & $13(3.2 \%)$ \\
\hline Sex with an HIV-positive partner & $6(1.5 \%)$ \\
\hline Do not engage in regular medical care & $154(38 \%)$ \\
\hline History of drug abuse & $134(33.1 \%)$ \\
\hline History of sex abuse & $30(7.4 \%)$ \\
\hline Used street drugs before sex & $128(31.6 \%)$ \\
\hline Sex with partner who exchanges sex for commodities & $49(12.1 \%)$ \\
\hline History of sexually transmitted infection & $25(6.2 \%)$ \\
\hline
\end{tabular}

Table 3: HIV Risk Factors $(N=405)$.

mention of prior sex abuse. Chart review revealed $33.1 \%$ of participants had a history of substance abuse, and $7.4 \%$ had a documented history of sexual abuse (Table 3).

\section{Discussion}

While more mentally ill patients are being tested for HIV compared to the rest of the population, over half of this high-risk group have never been tested, testing rates have been improving little over time, and routine screening is uncommon $[6,26]$. There remains a need for efforts to increase HIV testing rates amongst those with SMI and to effectively integrate routine screening programs into all mental health care settings. The success of Project B.R.I.E.F. in emergency departments has demonstrated the feasibility of expanding routine HIV screening to at-risk patients using rapid testing with facilitation and counseling by a dedicated, trained staff of Public Health Advocates [27]. This model effectively addresses many of the barriers to routine screening frequently cited by physicians, including inadequate time and training to offer screening and counseling, and the expectation of poor patient follow-up [25]. Utilizing a streamlined process of counseling, rapid testing and linkage to care allows for continued provision of HIV education and maximization of follow-up care for high-risk and newly diagnosed patients [27]. The results of this study show that a similar counselor-based HIV screening program can be effectively adapted and implemented in inpatient mental health care settings, allowing for the routine screening and counseling of a large number of high-risk, severely mentally ill patients.

Counselor-based HIV screening was found to be both practical on inpatient psychiatric units and acceptable to the majority of eligible inpatients. Of the patients determined to have capacity to consent, the Public Health Advocates were able to offer testing to nearly threequarters of them, limited primarily by some patients being unavailable at time of testing (which may indicate some bias in the data). Of the patients who were offered testing, $62.9 \%$ of them accepted. The acceptability was markedly lower than that previously seen in local emergency departments [28] as well as with screening efforts targeting outpatient mental health clinics, but was comparable to those targeting the more high-risk populations of dually diagnosed patients and psychiatric inpatients elsewhere [24,29,30]. This implies a possible inverse relationship between psychiatric symptom severity and testing acceptance, highlighting the need for improved education efforts with 
severely mentally ill patients. However, given that most eligible patients were able to be offered testing, and the majority of whom consented to participation, counselor-based screening proved to be an effective approach to offering routine testing even in the inpatient psychiatric setting, improving access for an especially high-risk subpopulation.

Of the patients who refused HIV testing, the reasons most frequently cited were feeling that they were not at risk (44.2\%), having been tested during the previous six months (25.6\%), already being diagnosed HIVpositive (11.6\%), fear that testing will slow treatment (4.65\%), and fear of being tested (4.65\%). That such a high percentage of patients who refused testing believed they were not at risk is of particular interest, as nearly half of patients with SMI have been shown to believe that they are at lower risk for contracting HIV than they are objectively found to be [14]. It is important to note, however, that refusal data was not collected for all patients due to staffing shortages. Therefore, these results may not be representative of the entire cohort. Despite this limitation, mentally ill patients have frequently been shown to have insufficient knowledge of HIV risk factors and modes of transmission, and to have significant misconceptions about HIV and AIDS [14-17]. Those who do have adequate knowledge of HIV tend to be patients at institutions where sexual health education is routine, and still engage in risky behavior despite their knowledge of HIV risk factors [14,17]. Clearly, patient education and counseling is a critical component of HIV screening for those with SMI, having the potential to improve HIV knowledge, link knowledge with personal risk factors and behavior modification, and increase testing acceptance rates. However, in an effort to minimize barriers to screening, the CDC recommends that such counseling be discontinued or provided separately from testing if necessary [1], and over $78 \%$ of outpatient psychiatric clinics in New York do not offer counseling [31]. The use of public health advocates in this counselor-based model of screening allowed for in-depth risk factor assessment and individualized pre- and post-test counseling to continue to be provided in conjunction with testing without creating any additional burden to mental health care providers.

Based on CDC criterion, $26.4 \%$ of those patients tested through our screening program were considered high-risk for HIV infection, engaging in either sex with multiple partners $(22.2 \%)$, injection drug use $(4.9 \%)$, sex trade $(4.9 \%)$, sex with a known injection drug user (3.2\%), or sex with a partner known to be HIV-positive (1.5\%). Many participants engaged in other risky sexual practices, including nearly one-third reporting that they used drugs prior to intercourse, and the small minority of participants reporting regular condom use. In their review of the risk factor literature, Meade and Sikemma found even higher rates of many of these risk behaviors; nearly half of SMI patients had multiple sex partners, close to one-fifth had engaged in prostitution, and another one-fifth in injection drug use, the majority of whom had also shared needles [11]. In addition to these welldocumented risk factors, a review of patients' charts revealed $33.1 \%$ of our study population had a history of drug abuse and $7.4 \%$ had a history of sex abuse. While the association between injection drug use, needle sharing and contraction of blood born pathogens is direct, use of non-injection drugs is associated with heightened risk for HIV contraction via risky sex behavior; those who use non-injection drugs have diminished inhibitions, are often more sexually active, involved in prostitution, have lower condom use and more commonly engage in anal sex [32]. A history of sex abuse is also substantially more common in patients with SMI, and is associated with an increase in high-risk sexual activity and further sexual assault $[11,33]$.

Despite so many patients being high-risk for HIV, 38\% did not have a primary care physician or receive regular medical care. As has been previously reported, patients with SMI receive significantly less medical care and face more delays in care than the general population, due primarily to poor accessibility $[34,35]$. Although individuals with SMI are some of the most at-risk for a variety of medical conditions, they are also some of the least likely to have access to medical care. Within the sizeable subpopulation not receiving regular care, mental health settings may serve as the primary source of preventive health care, health maintenance and health education. As such, De Hert et al. point to the necessity of integrated care and increasing the involvement of mental health care providers in the basic health monitoring and care of patients with SMI [36]. The integration of routine HIV screening into mental health settings can provide access to testing, education and an essential link to medical treatment for individuals who otherwise may experience significant delays in receiving each.

Of the 405 patients tested as part of the screening program, only one new diagnosis was made. While less than anticipated, 11.6\% (13 patients) of those who refused testing did so because they were already known to be HIV-positive, indicating a potential prevalence rate of over $4.3 \%$ in the consentable inpatient population (13 out of 294 patients). Again, the refusal reason for more than half of the patient population was unknown, a potential factor contributing to the lower than expected prevalence rate. That nearly $78 \%$ of the psychiatric inpatients self-reported having received HIV testing in the past is encouraging. If accurate, the high testing rate may reflect the success of recent organized efforts such as The Bronx Knows initiative, a Bronx-wide program from the New York City Department of Health which has significantly increased HIV testing in health care and community organizations since 2008 [37]. It may also signal a promising increase in awareness and routine screening since the CDC released its updated recommendations.

It is still alarming, however, that over one-fifth of the psychiatric inpatient participants had never been tested for HIV. Those experiencing more significant psychiatric symptoms may be at greatest risk for HIV infection [10]. Further, medical inpatients diagnosed with HIV frequently have higher viral loads, lower CD4+ cell counts and higher rates of AIDS diagnoses than those diagnosed as outpatients [18]. Given the lack of access those with SMI have to regular medical care, and that psychiatric symptoms may occur secondary to or be worsened by HIV infection [7], psychiatric inpatients are a population in particular need of HIV screening. Importantly, psychiatric patients have been found to have better medication adherence rates and survival than non-psychiatric patients when given antiretroviral therapy, the former being particularly evident with increased mental health care $[13,38]$. Routine screening of psychiatric inpatients for HIV provides an opportunity to reach a large population of high-risk patients who may not be tested elsewhere. Utilizing a counselor-based system in collaboration with mental health care providers such as described here, patient risk assessment and counseling can be efficiently provided alongside rapid HIV testing, and seropositive patients may be linked to care in conjunction with ongoing psychiatric treatment. This routine, integrated and collaborative approach may help to maximize health benefits for newly diagnosed patients.

Jacobi Medical Center is a public hospital serving largely lowto-moderate income patients, and over $80 \%$ of the study population was ethnic minorities. As such, the generalizability of our results is unknown. Despite this, our results may be helpful for populations that share demographic similarities to our sample. Finally, much of the data depended on patient understanding and self-report, as well as medical record completeness and accuracy, all of which are difficult to validate. 
Citation: Popiel MJ, Duvvi V, Turkieh A, Cowan E, Calderon Y, et al. (2016) Rapid Routine HIV Testing for Psychiatric Inpatients. J AIDS Clin Res 7: 555. doi:10.4172/2155-6113.1000555

\section{References}

1. Branson BM, Handsfield HH, Lampe MA, Janssen RS, Taylor AW, et al. (2006) Revised recommendations for HIV testing of adults, adolescents, and pregnant women in health-care settings. MMWR Recomm Rep 55: 1-17.

2. National HIVIAIDS Strategy: APA Recommendations for Prioritizing Mental and Behavioral Health in Federal Implementation Efforts (2010) American Psychological Association.

3. American Psychiatric Association. Position Statement on HIV Antibody Testing (2009) APA Official Actions.

4. Senn TE, Carey MP (2009) HIV testing among individuals with a severe mental illness: review, suggestions for research, and clinical implications. Psychol Med 39: $355-363$

5. Desai MM, Rosenheck RA, Desai RA (2007) Prevalence and correlates of human immunodeficiency virus testing and posttest counseling among outpatients with serious mental illness. J Nerv Ment Dis 195: 776-780.

6. Yehia BR, Cui W, Thompson WW, Zack MM, McKnight-Eily L, et al. (2014) HIV testing among adults with mental illness in the United States. AIDS Patient Care STDS 28: 628-634.

7. Uys H (2013) Prevalence and clinical presentation of HIV positive female psychiatric inpatients. Afr J Psychiatry (Johannesbg) 16: 23-28.

8. Rosenberg SD, Goodman LA, Osher FC, Swartz MS, Essock SM, et al. (2001) Prevalence of HIV, hepatitis $B$, and hepatitis $C$ in people with severe mental illness. Am J Public Health 91: 31-37.

9. Blank MB, Mandell DS, Aiken L, Hadley TR (2002) Co-occurrence of HIV and serious mental illness among Medicaid recipients. Psychiatr Serv 53: 868-873.

10. Blank MB, Himelhoch SS, Balaji AB, Metzger DS, Dixon LB, et al. (2014) A multisite study of the prevalence of HIV with rapid testing in mental health settings. Am J Public Health 104: 2377-2384.

11. Meade CS, Sikkema KJ (2005) HIV risk behavior among adults with severe mental illness: a systematic review. Clin Psychol Rev 25: 433-457.

12. Prince JD, Walkup J, Akincigil A, Amin S, Crystal S (2012) Serious mental illness and risk of new HIVIAIDS diagnoses: an analysis of Medicaid beneficiaries in eight states. Psychiatr Serv 63: 1032-1038.

13. Himelhoch S, McCarthy JF, Ganoczy D, Medoff D, Dixon LB, et al. (2007) Understanding associations between serious mental illness and HIV among patients in the VA Health System. Psychiatr Serv. 58: 1165-1172.

14. Knox MD, Boaz TL, Friedrich MA, Dow MG (1994) HIV risk factors for persons with serious mental illness. Community Ment Health J 30: 551-563.

15. Ngwena J (2011) HIVIAIDS awareness in those diagnosed with mental illness. J Psychiatr Ment Health Nurs 18: 213-220.

16. Grassi L, Pavanati M, Cardelli R, Ferri S, Peron L (1999) HIV-risk behaviou and knowledge about HIVIAIDS among patients with schizophrenia. Psychol Med 29: 171-179.

17. Magagula TG, Mamabolo MM, Krüger C, Fletcher L (2012) A survey of HIVrelated knowledge among adult psychiatric patients. A South African Study Part 2. Afr J Psychiatry (Johannesbg). 15: 335-339.

18. Greenwald JL, Rich CA, Bessega S, Posner MA, Maeda JL, et al. (2006) Evaluation of the Centers for Disease Control and Prevention's recommendations regarding routine testing for human immunodeficiency virus by an inpatient service: who are we missing? Mayo Clin Proc. 81: 452-458.

19. Owens DK, Sundaram V, Lazzeroni LC, Douglass L, Sanders GD et al. (2007) Prevalence of HIV infection among inpatients and outpatients in Department of Veterans Affairs health care systems: implications for screening programs for HIV. Am J Public Health 97: 2173-2178.

20. Jain CL, Jue JS, MacKay R, Wallach F, Factor SH, et al. (2008) Acceptance of rapid HIV testing among medical inpatients in New York City. AIDS Patient Care STDS 22: 657-662.

21. Scott VF, Sitapati A, Martin S, Summers P, Washington M, et al. (2009) The Howard University Hospital experience with routineized HIV screening: a progress report. Trans Am Clin Climatol Assoc 120: 429-434.

22. Siegel M, Kennedy L, Rexroth K, Lankford M, Turner M, et al. (2010) Better but not ideal acceptance of routine inpatient HIV point-of-care testing among veterans in a high prevalence area. J Acquir Immune Defic Syndr 55: 205-210.
23. Higginbotham S, Holmes R, Stone H, Beil J, Datu GB, et al. (2000) Adoption of protective behaviors among persons with recent HIV infection and diagnosis - Alabama, New Jersey, and Tennessee, 1997- 1998. Journal of the American Medical Association 284: 171-173.

24. Sanger C, Hayward J, Patel G, Phekoo K, Poots AJ, et al. (2013) Acceptability and necessity of HIV and other blood-borne virus testing in a psychiatric setting. Br J Psychiatry 202: 307-308.

25. Burke RC, Sepkowitz KA, Bernstein KT, Karpati AM, Myers JE, et al. (2007) Why don't physicians test for HIV? A review of the US literature. AIDS 21: 16171624 .

26. Blumberg SJ, Dickey WC (2003) Prevalence of HIV risk behaviors, risk perceptions, and testing among US adults with mental disorders. J Acquir Immune Defic Syndr 32: 77-79.

27. Calderon Y, Leider J, Hailpern S, Chin R, Ghosh R, et al. (2009) High-volume rapid HIV testing in an urban emergency department. AIDS Patient Care STDS 23: 749-755.

28. Sanders GD, Anaya HD, Asch S, Hoang T, Golden JF, et al. (2010) Costeffectiveness of strategies to improve HIV testing and receipt of results: economic analysis of a randomized controlled trial. J Gen Intern Med 25: 556563.

29. Rosenberg SD, Goldberg RW, Dixon LB, Wolford GL, Slade EP, et al. (2010) Assessing the STIRR model of best practices for blood-borne infections of clients with severe mental illness. Psychiatr Serv 61: 885-891.

30. Rosenberg S, Brunette M, Oxman T, Marsh B, Dietrich A, et al. (2004) The STIRR model of best practices for blood-borne diseases among clients with serious mental illness. Psychiatr Serv 55: 660-664.

31. Satriano J, McKinnon K, Adoff S (2007) HIV service provision for people with severe mental illness in outpatient mental health care settings in New York. J Prev Interv Community 33: 95-108.

32. Khan MR, Berger A, Hemberg J, O'Neill A, Dyer TP, et al. (2013) Non-injection and injection drug use and STI/HIV risk in the United States: the degree to which sexual risk behaviors versus sex with an STI-infected partner account for infection transmission among drug users. AIDS Behav. 17: 1185-1194.

33. Meade CS, Kershaw TS, Hansen NB, Sikkema KJ (2009) Long-term correlates of childhood abuse among adults with severe mental illness: adult victimization, substance abuse, and HIV sexual risk behavior. AIDS Behav 13: 207-216.

34. Bradford DW, Kim MM, Braxton LE, Marx CE, Butterfield M, et al. (2008) Access to medical care among persons with psychotic and major affective disorders. Psychiatr Serv 59: 847-852.

35. Mojtabai R, Cullen B, Everett A, Nugent KL, Sawa A, et al. (2014) Reasons for not seeking general medical care among individuals with serious mental illness. Psychiatr Serv 65: 818-821.

36. De Hert M, Cohen D, Bobes J, Cetkovich-Bakmas M, Leucht S et al. (2011) Physical illness in patients with severe mental disorders. II. Barriers to care monitoring and treatment guidelines, plus recommendations at the system and individual level. World Psychiatry 10: 138-151.

37. New York City Department of Health and Mental Hygiene (2011) The Bronx Knows HIV Testing Initiative Final Report.

38. Himelhoch S, Moore RD, Treisman G, Gebo KA (2004) Does the presence of a current psychiatric disorder in AIDS patients affect the initiation of antiretroviral treatment and duration of therapy? J Acquir Immune Defic Syndr. 37: 14571463 